\title{
Relaxed inertial accelerated algorithms for solving split equality feasibility problem
}

\author{
Meixia Lia,*, Xiping Kao ${ }^{\mathrm{b}}$, Haitao $\mathrm{Che}^{\mathrm{a}}$ \\ a School of Mathematics and Information Science, Weifang University, Weifang, 261061, China. \\ ${ }^{b}$ College of Mathematics and Systems Science, Shandong University of Science and Technology, Qingdao, 266590, China. \\ Communicated by S. S. Chang
}

\begin{abstract}
In this paper, we study the split equality feasibility problem and present two algorithms for solving the problem with special structure. We prove the weak convergence of these algorithms under mild conditions. Especially, the selection of stepsize is only dependent on the information of current iterative points, but independent from the prior knowledge of operator norms. These algorithms provide new ideas for solving the split equality feasibility problem. Numerical results demonstrate the feasibility and effectiveness of these algorithms. (c)2017 All rights reserved.
\end{abstract}

Keywords: Split equality feasibility problem, relaxed inertial accelerated algorithm, weak convergence, subdifferential. 2010 MSC: 47H09, 65K05, 47J25.

\section{Introduction}

Let $\mathrm{H}_{1}, \mathrm{H}_{2}$, and $\mathrm{H}_{3}$ be real Hilbert spaces with inner product $\langle\cdot, \cdot\rangle$ and norm $\|\cdot\|$, and $\mathrm{C}$ and $\mathrm{Q}$ be nonempty closed convex subsets of $\mathrm{H}_{1}$ and $\mathrm{H}_{2}$, respectively. Assume that $A: \mathrm{H}_{1} \rightarrow \mathrm{H}_{3}$ and $B: \mathrm{H}_{2} \rightarrow \mathrm{H}_{3}$ be two bounded linear operators. The split equality feasibility problem (SEFP) is to find

$$
x \in C, y \in Q \text { such that } A x=B y,
$$

which allows asymmetric and partial relations between the variables $x$ and $y$. The problem can be used in many situations, for instance applications in decomposition methods for PDE's, in game theory and in intensity-modulated radiation therapy.

In recent years, the SEFP attracted many researchers to study its algorithms and applications. Especially, when SEFP (1.1) has at least one solution, it can be converted to the following fixed point equation system

$$
\left\{\begin{array}{l}
x=P_{C}\left(x-\gamma A^{*}(A x-B y)\right) \\
y=P_{Q}\left(y+\gamma B^{*}(A x-B y)\right)
\end{array}\right.
$$

\footnotetext{
${ }^{*}$ Corresponding author

Email addresses: limeixia001@163.com (Meixia Li), 805357576@qq.com (Xiping Kao), haitaoche@163. com (Haitao Che)
} doi:10.22436/jnsa.010.08.07 
where $\gamma$ is a positive constant. According to (1.2), Byrne and Moudafi [2] proposed and proved the convergence of the following projection gradient algorithm

$$
\left\{\begin{array}{l}
x_{k}=P_{C}\left(x_{k}-\gamma_{k} A^{*}\left(A x_{k}-B y_{k}\right)\right) \\
y_{k}=P_{Q}\left(y_{k}+\gamma_{k} B^{*}\left(A x_{k}-B y_{k}\right)\right)
\end{array}\right.
$$

where $\gamma_{k} \in\left(\epsilon, \min \left(\frac{1}{\lambda_{A}}, \frac{1}{\lambda_{B}}\right)-\epsilon\right), \epsilon$ is a small enough positive constant, and $\lambda_{A}, \lambda_{B}$ are the spectral radiuses of $A^{*} A$ and $B * B$, respectively. Then, Moudifi [15] presented and studied the convergence of the following alternating $C Q$-algorithm

$$
\left\{\begin{array}{l}
x_{k}=P_{C}\left(x_{k}-\gamma_{k} A^{*}\left(A x_{k}-B y_{k}\right)\right) \\
y_{k}=P_{Q}\left(y_{k}+\gamma_{k} B^{*}\left(A x_{k+1}-B y_{k}\right)\right)
\end{array}\right.
$$

where it is needed that $\gamma_{k}$ be positive no-decreasing sequence except for the above conditions. Further, a lot of researchers studied the algorithms for solving SEFP (1.1) and proved their convergence, for details see $[6,7,15]$ and references therein.

Note that, by taking $B=I$ in (1.1), the problem is converted to convex feasibility problem (CFP), that is to find

$$
x \in C \text { such that } A x \in Q \text {, }
$$

which is originally introduced by Censor and Elfving [4] and has broad applications in many fields such as image reconstruction problem [3, 13], approximation theory [10], control [12], and so on. About the study of algorithm and theory for solving CFP (1.3), readers can see [17, 19-22], etc.. Recently, Dang et al. [9] proposed two inertial accelerated algorithms to solve CFP (1.3) and proved their asymptotical convergence under some conditions, for example, the selection of stepsize is dependent on the spectral radius of $A^{*} A$.

Inspired by the related references, we study SEFP (1.1) and propose two algorithms for the special structure of $C$ and $Q$. We prove that the sequences generated by these algorithms weakly converge to the solution of (1.1) under suitable conditions. Especially, the selection of stepsize is only determined by the information of current iterative points, without the prior knowledge of operator norms. Numerical results illustrate the feasibility and effectiveness of these algorithms. At the same time, these algorithms provide new ideas for solving the split equality feasibility problem.

The rest of this paper is organized as follows. In Section 2, we review some basic concepts and lemmas. In Section 3, we present two algorithms, denoted by the relaxed inertial accelerated algorithm and the modified relaxed inertial accelerated algorithm, and prove their convergence. In Section 4, we demonstrate the feasibility and effectiveness of these algorithms through some numerical examples.

\section{Preliminaries}

In this section, we introduce some concepts and conclusions which are needed in the following. Let $\mathrm{H}$ be a Hilbert space. A mapping $\mathrm{T}: \mathrm{H} \rightarrow \mathrm{H}$ is called contraction, if there exists a constant $\alpha \in(0,1)$ such that

$$
\|T x-T y\| \leqslant \alpha\|x-y\|, \quad \forall x, y \in H .
$$

If $\alpha=1$, then $T$ is called nonexpansive.

A mapping $P_{C}$ is said to be metric projection of $H$ onto $C$ if for every point $x \in H$, there exists a unique nearest point in $\mathrm{C}$ denoted by $\mathrm{P}_{\mathrm{C}} \times$ such that

$$
\left\|x-P_{C} x\right\| \leqslant\|x-y\|, \quad \forall y \in C .
$$

It is well-known that $\mathrm{P}_{\mathrm{C}}$ is a nonexpansive mapping and is characterized by the following properties

$$
\begin{aligned}
\left\|P_{C} x-P_{C} y\right\|^{2} & \leqslant\left\langle x-y, P_{C} x-P_{C} y\right\rangle, \quad \forall x, y \in H, \\
\left\langle x-P_{C} x, y-P_{C} x\right\rangle & \leqslant 0, \quad \forall x \in H, y \in C,
\end{aligned}
$$




$$
\|x-y\|^{2} \geqslant\left\|x-P_{C} x\right\|^{2}+\left\|y-P_{C} x\right\|^{2}, \quad \forall x \in H, y \in C,
$$

and

$$
\left\|(x-y)-\left(P_{C} x-P_{C} y\right)\right\|^{2} \geqslant\|x-y\|^{2}-\left\|P_{C} x-P_{C} y\right\|^{2}, \quad \forall x, y \in H .
$$

Definition 2.1 ([8]). Let $f: H \rightarrow R$ be appropriate convex. The subdifferential of $f$ at $x$ is defined as

$$
\partial f(x)=\{\xi \in H \mid f(y) \geqslant f(x)+\langle\xi, y-x\rangle, \quad \forall y \in H\} .
$$

Lemma $2.2([14])$. Assume $\varphi_{\mathrm{k}} \in[0, \infty)$ and $\delta_{\mathrm{k}} \in[0, \infty)$ satisfy

(a) $\varphi_{k+1}-\varphi_{k} \leqslant \theta_{k}\left(\varphi_{k}-\varphi_{k-1}\right)+\delta_{k}$;

(b) $\sum_{k=1}^{\infty} \delta_{k}<\infty$;

(c) $\left\{\theta_{\mathrm{k}}\right\} \subset[0, \theta]$, where $\theta \in[0,1)$.

Then, the sequence $\left\{\varphi_{\mathrm{k}}\right\}$ is convergent with $\sum_{k=1}^{\infty}\left[\varphi_{\mathrm{k}+1}-\varphi_{\mathrm{k}}\right]_{+}<\infty$, where $t_{+}:=\max \{\mathrm{t}, 0\}$ for any $\mathrm{t} \in \mathrm{R}$.

Lemma 2.3 ([5]). If $x, y, z \in \mathrm{H}$, then

(a) $\|x+y\|^{2} \leqslant\|x\|^{2}+2\langle y, x+y\rangle$;

(b) for any $\lambda \in[0,1]$,

$$
\|\lambda x+(1-\lambda) y\|^{2}=\lambda\|x\|^{2}+(1-\lambda)\|y\|^{2}-\lambda(1-\lambda)\|x-y\|^{2} ;
$$

(c) for $\mathrm{a}, \mathrm{b}, \mathrm{c} \in[0,1]$ with $\mathrm{a}+\mathrm{b}+\mathrm{c}=1$,

$$
\|a x+b y+c z\|^{2}=a\|x\|^{2}+b\|y\|^{2}+c\|z\|^{2}-a b\|x-y\|^{2}-a c\|x-z\|^{2}-b c\|y-z\|^{2} .
$$

\section{Main results}

In this section, we give the main results of this paper. First, we describe the algorithm for solving SEFP (1.1).

Throughout the rest of this paper, we assume that $\mathrm{C}$ and $\mathrm{Q}$ are denoted as

$$
\mathrm{C}=\left\{x \in \mathrm{H}_{1} \mid \mathrm{c}(\mathrm{x}) \leqslant 0\right\}
$$

and

$$
\mathrm{Q}=\left\{\mathrm{y} \in \mathrm{H}_{2} \mid \mathrm{q}(\mathrm{y}) \leqslant 0\right\},
$$

where $c: H_{1} \rightarrow R$ and $q: H_{2} \rightarrow R$ are appropriate convex and $C, Q$ are nonempty. We denote and assume

$$
\Gamma=\{(x, y) \mid x \in C, y \in Q \text { such that } A x=B y\} \neq \emptyset,
$$

where C, Q are defined as (3.1) and (3.2), respectively.

Furthermore, we assume that there exists at least one subgradient $\xi \in \partial c(x)$ for any $x \in \mathrm{H}_{1}$ and one subgradient $\eta \in \partial q(y)$ for any $y \in H_{2}$ such that they all can be calculated. And all $\xi \in \partial c(x)$ for any $x \in H_{1}$ and all $\eta \in \partial q(y)$ for any $y \in H_{2}$ are bounded in any bounded subsets of $H_{1}$ and $H_{2}$, respectively. Define two sets at point $x^{k}$ and $y^{k}$, respectively,

$$
\mathrm{C}_{\mathrm{k}}=\left\{x \in \mathrm{H}_{1} \mid \mathrm{c}\left(\mathrm{x}^{\mathrm{k}}\right)+\left\langle\xi^{\mathrm{k}}, x-x^{\mathrm{k}}\right\rangle \leqslant 0\right\}
$$

where $\xi^{k} \in \partial c\left(x^{k}\right)$, and

where $\eta^{k} \in \partial q\left(y^{k}\right)$.

$$
Q_{k}=\left\{y \in H_{2} \mid q\left(y^{k}\right)+\left\langle\eta^{k}, y-y^{k}\right\rangle \leqslant 0\right\}
$$

It is easy to see that the halfspaces $C_{k}$ and $Q_{k}$ contain $C$ and $Q$, respectively, according to the definition of subgradient. And the orthogonal projections onto $C_{k}$ and $Q_{k}$ can be easily determined, for details see $[1,11,16]$.

Now we state the relaxed inertial accelerated algorithm. 


\section{Algorithm 3.1.}

Initialization: Take $x^{0}, x^{1} \in \mathrm{H}_{1}, \mathrm{y}^{0}, \mathrm{y}^{1} \in \mathrm{H}_{2}$ arbitrarily.

Iterative step: For $k \geqslant 1$, given the points $x^{k}, x^{k-1}, y^{k}, y^{k-1}$, the next iterative points $x^{k+1}, y^{k+1}$ are generated by

$$
\left\{\begin{array}{l}
u^{k}=x^{k}+\theta_{k}\left(x^{k}-x^{k-1}\right) \\
v^{k}=y^{k}+\theta_{k}\left(y^{k}-y^{k-1}\right) \\
x^{k+1}=P_{C_{k}}\left(u^{k}-\gamma_{k} A^{*}\left(A u^{k}-B v^{k}\right)\right) \\
y^{k+1}=P_{Q_{k}}\left(v^{k}+\gamma_{k} B^{*}\left(A u^{k}-B v^{k}\right)\right)
\end{array}\right.
$$

where $\theta_{k} \in[0,1), \gamma_{k}$ is positive stepsize, and $C_{k}, Q_{k}$ are defined as (3.3) and (3.4), respectively.

As follows, we give the convergence theorem of Algorithm 3.1.

Theorem 3.2. For any $k \geqslant 1$, choose parameter $\theta_{k} \in\left[0, \bar{\theta}_{k}\right]$ with $\bar{\theta}_{k}=\min \left\{\theta,\left(\max \left\{k^{2}\left\|x^{k}-x^{k-1}\right\|^{2}, k^{2} \| x^{k}-\right.\right.\right.$ $\left.\left.\left.x^{k-1}\left\|, k^{2}\right\| y^{k}-y^{k-1}\left\|^{2}, k^{2}\right\| y^{k}-y^{k-1} \|\right\}\right)^{-1}\right\}$, where $\theta \in[0,1)$ and

$$
\gamma_{k} \in\left(\epsilon, \min \left\{\delta,(1-\sigma) \frac{2\left\|A u^{k}-B v^{k}\right\|^{2}}{\left\|A^{*}\left(A u^{k}-B v^{k}\right)\right\|^{2}+\left\|B^{*}\left(A u^{k}-B v^{k}\right)\right\|^{2}}\right\}\right),
$$

where $\epsilon>0$ is small enough, $\sigma \in(0,1)$, and $\delta>\epsilon$. Then the sequence $\left\{\left(x^{k}, y^{k}\right)\right\}$ generated by Algorithm 3.1 converges weakly to a solution $(x, y)$ of (1.1).

Proof. If $\theta_{k} \equiv 0$, the conclusion is true from [2].

Now, we assume that $\theta_{k}>0$ for some $k \in N$. Let $\left(x^{*}, y^{*}\right)$ be the solution of (1.1). Since $C \subset C_{k}, Q \subset$ $\mathrm{Q}_{k}$, then

$$
x^{*}=\mathrm{P}_{\mathrm{C}}\left(x^{*}\right)=\mathrm{P}_{\mathrm{C}_{\mathrm{k}}}\left(\mathrm{x}^{*}\right), \quad \mathrm{y}^{*}=\mathrm{P}_{\mathrm{Q}}\left(\mathrm{y}^{*}\right)=\mathrm{P}_{\mathrm{Q}_{\mathrm{k}}}\left(\mathrm{y}^{*}\right) .
$$

Define two auxiliary real sequences $\varphi_{k}=\frac{1}{2}\left\|x^{k}-x^{*}\right\|^{2}$ and $\psi_{k}=\frac{1}{2}\left\|y^{k}-y^{*}\right\|^{2}$. According to the nonexpansive property of the operators $\mathrm{P}_{\mathrm{C}}, \mathrm{P}_{\mathrm{Q}}$ and (3.5), one has

$$
\begin{aligned}
\varphi_{k+1}= & \frac{1}{2}\left\|x^{k+1}-x^{*}\right\|^{2} \\
= & \frac{1}{2}\left\|P_{C_{k}}\left(u^{k}-\gamma_{k} A^{*}\left(A u^{k}-B v^{k}\right)\right)-P_{C_{k}}\left(x^{*}\right)\right\|^{2} \\
\leqslant & \frac{1}{2}\left\|u^{k}-\gamma_{k} A^{*}\left(A u^{k}-B v^{k}\right)-x^{*}\right\|^{2} \\
= & \frac{1}{2}\left\|u^{k}-x^{*}\right\|^{2}+\frac{1}{2} \gamma_{k}^{2}\left\|A^{*}\left(A u^{k}-B v^{k}\right)\right\|^{2}-\gamma_{k}\left\langle u^{k}-x^{*}, A^{*}\left(A u^{k}-B v^{k}\right)\right\rangle \\
= & \frac{1}{2}\left\|u^{k}-x^{*}\right\|^{2}+\frac{1}{2} \gamma_{k}^{2}\left\|A^{*}\left(A u^{k}-B v^{k}\right)\right\|^{2}-\gamma_{k}\left\langle A u^{k}-B v^{k}, A u^{k}-B v^{k}\right\rangle \\
& -\gamma_{k}\left\langle B v^{k}-A x^{*}, A u^{k}-B v^{k}\right\rangle \\
= & \frac{1}{2}\left\|u^{k}-x^{*}\right\|^{2}+\frac{1}{2} \gamma_{k}^{2}\left\|A^{*}\left(A u^{k}-B v^{k}\right)\right\|^{2}-\gamma_{k}\left\|A u^{k}-B v^{k}\right\|^{2}-\gamma_{k}\left\langle B v^{k}-A x^{*}, A u^{k}-B v^{k}\right\rangle .
\end{aligned}
$$

Furthermore,

$$
\begin{aligned}
\frac{1}{2}\left\|u^{k}-x^{*}\right\|^{2} & =\frac{1}{2}\left\|x^{k}+\theta_{k}\left(x^{k}-x^{k-1}\right)-x^{*}\right\|^{2} \\
& =\frac{1}{2}\left\|x^{k}-x^{*}\right\|^{2}+\theta_{k}\left\langle x^{k}-x^{*}, x^{k}-x^{k-1}\right\rangle+\frac{\theta_{k}^{2}}{2}\left\|x^{k}-x^{k-1}\right\|^{2}
\end{aligned}
$$

and

$$
\begin{aligned}
\frac{1}{2}\left\|x^{k}-x^{*}\right\|^{2} & =\frac{1}{2}\left\|x^{k}-x^{k-1}+x^{k-1}-x^{*}\right\|^{2} \\
& =\frac{1}{2}\left\|x^{k-1}-x^{*}\right\|^{2}+\left\langle x^{k}-x^{k-1}, x^{k-1}-x^{*}\right\rangle+\frac{1}{2}\left\|x^{k}-x^{k-1}\right\|^{2} \\
& =\frac{1}{2}\left\|x^{k-1}-x^{*}\right\|^{2}+\left\langle x^{k}-x^{k-1}, x^{k-1}-x^{k}+x^{k}-x^{*}\right\rangle+\frac{1}{2}\left\|x^{k}-x^{k-1}\right\|^{2} \\
& =\frac{1}{2}\left\|x^{k-1}-x^{*}\right\|^{2}+\left\langle x^{k}-x^{k-1}, x^{k}-x^{*}\right\rangle-\frac{1}{2}\left\|x^{k}-x^{k-1}\right\|^{2} .
\end{aligned}
$$


Combining (3.8) with (3.9), we obtain

$$
\frac{1}{2}\left\|u^{k}-x^{*}\right\|^{2}=\varphi_{k}+\theta_{k}\left(\varphi_{k}-\varphi_{k-1}\right)+\frac{\theta_{k}+\theta_{k}^{2}}{2}\left\|x^{k}-x^{k-1}\right\|^{2}
$$

From (3.10) and (3.7), one has

$$
\begin{aligned}
\varphi_{k+1} \leqslant & \varphi_{k}+\theta_{k}\left(\varphi_{k}-\varphi_{k-1}\right)+\frac{\theta_{k}+\theta_{k}^{2}}{2}\left\|x^{k}-x^{k-1}\right\|^{2}+\frac{1}{2} \gamma_{k}^{2}\left\|A^{*}\left(A u^{k}-B v^{k}\right)\right\|^{2} \\
& -\gamma_{k}\left\|A u^{k}-B v^{k}\right\|^{2}-\gamma_{k}\left\langle B v^{k}-A x^{*}, A u^{k}-B v^{k}\right\rangle .
\end{aligned}
$$

Analogous to the proof of (3.7)-(3.11), we can obtain

$$
\begin{aligned}
\psi_{k+1}=\frac{1}{2}\left\|y^{k+1}-y^{*}\right\|^{2} & \leqslant \frac{1}{2}\left\|v^{k}+\gamma_{k} B^{*}\left(A u^{k}-B v^{k}\right)-y^{*}\right\|^{2} \\
& =\frac{1}{2}\left\|v^{k}-y^{*}\right\|^{2}+\frac{1}{2} \gamma_{k}^{2}\left\|B^{*}\left(A u^{k}-B v^{k}\right)\right\|^{2}+\gamma_{k}\left\langle A u^{k}-B v^{k}, B v^{k}-B y^{*}\right\rangle, \\
\frac{1}{2}\left\|v^{k}-y^{*}\right\|^{2} & =\frac{1}{2}\left\|y^{k}+\theta_{k}\left(y^{k}-y^{k-1}\right)-y^{*}\right\|^{2} \\
& =\frac{1}{2}\left\|y^{k}-y^{*}\right\|^{2}+\theta_{k}\left\langle y^{k}-y^{*}, y^{k}-y^{k-1}\right\rangle+\frac{\theta_{k}^{2}}{2}\left\|y^{k}-y^{k-1}\right\|^{2},
\end{aligned}
$$

and

$$
\psi_{k}=\frac{1}{2}\left\|y^{k}-y^{*}\right\|^{2}=\frac{1}{2}\left\|y^{k}-y^{k-1}+y^{k-1}-y^{*}\right\|^{2}=\psi_{k-1}+\left\langle y^{k}-y^{k-1}, y^{k}-y^{*}\right\rangle-\frac{1}{2}\left\|y^{k}-y^{k-1}\right\|^{2},
$$

which implies that

$$
\left\langle y^{k}-y^{k-1}, y^{k}-y^{*}\right\rangle=\psi_{k}-\psi_{k-1}+\frac{1}{2}\left\|y^{k}-y^{k-1}\right\|^{2}
$$

Combining (3.13) with (3.14), we have

$$
\frac{1}{2}\left\|v^{k}-y^{*}\right\|^{2}=\psi_{k}+\theta_{k}\left(\psi_{k}-\psi_{k-1}\right)+\frac{\theta_{k}+\theta_{k}^{2}}{2}\left\|y^{k}-y^{k-1}\right\|^{2}
$$

From (3.12) and (3.15), we obtain

$$
\begin{aligned}
\psi_{k+1} \leqslant & \psi_{k}+\theta_{k}\left(\psi_{k}-\psi_{k-1}\right)+\frac{\theta_{k}+\theta_{k}^{2}}{2}\left\|y^{k}-y^{k-1}\right\|^{2}+\frac{1}{2} \gamma_{k}^{2}\left\|B^{*}\left(A u^{k}-B v^{k}\right)\right\|^{2} \\
& +\gamma_{k}\left\langle B v^{k}-B y^{*}, A u^{k}-B v^{k}\right\rangle .
\end{aligned}
$$

According to (3.11) and (3.16) as well as $A x^{*}=B y^{*}$, one has

$$
\begin{aligned}
\varphi_{k+1}+\psi_{k+1} \leqslant & \varphi_{k}+\psi_{k}+\theta_{k}\left(\varphi_{k}+\psi_{k}-\varphi_{k-1}-\psi_{k-1}\right)+\frac{\theta_{k}+\theta_{k}^{2}}{2}\left(\left\|x^{k}-x^{k-1}\right\|^{2}+\left\|y^{k}-y^{k-1}\right\|^{2}\right) \\
& +\frac{1}{2} \gamma_{k}^{2}\left(\left\|A^{*}\left(A u^{k}-B v^{k}\right)\right\|^{2}+\left\|B^{*}\left(A u^{k}-B v^{k}\right)\right\|^{2}\right)-\gamma_{k}\left\|A u^{k}-B v^{k}\right\|^{2} .
\end{aligned}
$$

From (3.6), we have

$$
\frac{1}{2} \gamma_{k}^{2}\left(\left\|A^{*}\left(A u^{k}-B v^{k}\right)\right\|^{2}+\left\|B^{*}\left(A u^{k}-B v^{k}\right)\right\|^{2}\right)-\gamma_{k}\left\|A u^{k}-B v^{k}\right\|^{2} \leqslant-\sigma \gamma_{k}\left\|A u^{k}-B v^{k}\right\|^{2} .
$$

According to the definition of $\theta_{k}$, we know that

$$
\theta_{k}^{2} \leqslant \theta_{k}, \quad \theta_{k}\left\|x^{k}-x^{k-1}\right\|^{2} \leqslant \frac{1}{k^{2}}, \quad \theta_{k}\left\|y^{k}-y^{k-1}\right\|^{2} \leqslant \frac{1}{k^{2}}
$$


From (3.17)-(3.19), we can deduce that

$$
\left(\varphi_{k+1}+\psi_{k+1}\right)-\left(\varphi_{k}+\psi_{k}\right) \leqslant \theta_{k}\left(\left(\varphi_{k}+\psi_{k}\right)-\left(\varphi_{k-1}+\psi_{k-1}\right)\right)+\theta_{k}\left(\left\|x^{k}-x^{k-1}\right\|^{2}+\left\|y^{k}-y^{k-1}\right\|^{2}\right)
$$

and

$$
\sum_{k=1}^{\infty} \theta_{k}\left(\left\|x^{k}-x^{k-1}\right\|^{2}+\left\|y^{k}-y^{k-1}\right\|^{2}\right)<\infty .
$$

From Lemma 2.2, the sequence $\left\{\varphi_{k}+\psi_{k}\right\}$ is convergent with

$$
\sum_{k=1}^{\infty}\left[\varphi_{k+1}+\psi_{k+1}-\varphi_{k}-\psi_{k}\right]_{+}<\infty,
$$

where $[t]_{+}=\max \{t, 0\}$ for any $t \in R$. Therefore, $\left\{x^{k}\right\}$ and $\left\{y^{k}\right\}$ are bounded, so are $\left\{u^{k}\right\}$ and $\left\{v^{k}\right\}$. By (3.17), (3.18), and $\theta_{k}^{2} \leqslant \theta_{k}<1$, we have

$$
\sum_{k=1}^{\infty}\left\|A u^{k}-B v^{k}\right\|<\infty
$$

Thus,

$$
\lim _{k \rightarrow \infty}\left\|A u^{k}-B v^{k}\right\|=0
$$

Next, we show that

$$
\lim _{k \rightarrow \infty}\left\|x^{k+1}-x^{k}\right\|=0 \quad \text { and } \quad \lim _{k \rightarrow \infty}\left\|y^{k+1}-y^{k}\right\|=0
$$

In fact,

$$
\begin{aligned}
\left\|x^{k+1}-x^{*}\right\|^{2} & =\left\|\left(x^{k+1}-u^{k}\right)+\left(u^{k}-x^{*}\right)\right\|^{2} \\
& =\left\|x^{k+1}-u^{k}\right\|^{2}+\left\|u^{k}-x^{*}\right\|^{2}+2\left\langle x^{k+1}-u^{k}, u^{k}-x^{*}\right\rangle \\
& =\left\|x^{k+1}-u^{k}\right\|^{2}+\left\|u^{k}-x^{*}\right\|^{2}+2\left\langle x^{k+1}-u^{k}, u^{k}-x^{k+1}\right\rangle+2\left\langle x^{k+1}-u^{k}, x^{k+1}-x^{*}\right\rangle
\end{aligned}
$$

and

$$
\left\|x^{k+1}-u^{k}\right\|^{2}=\left\|u^{k}-x^{*}\right\|^{2}-\left\|x^{k+1}-x^{*}\right\|^{2}+2\left\langle x^{k+1}-u^{k}, x^{k+1}-x^{*}\right\rangle .
$$

Combining (3.10), (3.23) with the definition of $\varphi_{k}$, we have

$$
\begin{aligned}
\left\|x^{k+1}-u^{k}\right\|^{2}= & \left\|x^{k}-x^{*}\right\|^{2}-\left\|x^{k+1}-x^{*}\right\|^{2}+\theta_{k}\left(\left\|x^{k}-x^{*}\right\|^{2}-\left\|x^{k-1}-x^{*}\right\|^{2}\right) \\
& +\left(\theta_{k}+\theta_{k}^{2}\right)\left\|x^{k}-x^{k-1}\right\|^{2}+2\left\langle x^{k+1}-u^{k}, x^{k+1}-x^{*}\right\rangle .
\end{aligned}
$$

Similarly, we can lead to

$$
\begin{aligned}
\left\|y^{k+1}-v^{k}\right\|^{2}= & \left\|y^{k}-y^{*}\right\|^{2}-\left\|y^{k+1}-y^{*}\right\|^{2}+\theta_{k}\left(\left\|y^{k}-y^{*}\right\|^{2}-\left\|y^{k-1}-y^{*}\right\|^{2}\right) \\
& +\left(\theta_{k}+\theta_{k}^{2}\right)\left\|y^{k}-y^{k-1}\right\|^{2}+2\left\langle y^{k+1}-v^{k}, y^{k+1}-y^{*}\right\rangle .
\end{aligned}
$$

By (3.24) and (3.25), one has

$$
\begin{aligned}
\left\|x^{k+1}-u^{k}\right\|^{2}+\left\|y^{k+1}-v^{k}\right\|^{2} \leqslant & \left(\left\|x^{k}-x^{*}\right\|^{2}+\left\|y^{k}-y^{*}\right\|^{2}-\left\|x^{k+1}-x^{*}\right\|^{2}-\left\|y^{k+1}-y^{*}\right\|^{2}\right)_{+} \\
& +\theta_{k}\left(\left\|x^{k}-x^{*}\right\|^{2}+\left\|y^{k}-y^{*}\right\|^{2}-\left\|x^{k-1}-x^{*}\right\|^{2}-\left\|y^{k-1}-y^{*}\right\|^{2}\right)_{+} \\
& +2 \theta_{k}\left(\left\|x^{k}-x^{k-1}\right\|^{2}+\left\|y^{k}-y^{k-1}\right\|^{2}\right)+2\left\langle x^{k+1}-u^{k}, x^{k+1}-x^{*}\right\rangle \\
& +2\left\langle y^{k+1}-v^{k}, y^{k+1}-y^{*}\right\rangle .
\end{aligned}
$$


On the other hand, from (3.5) and (2.1), we obtain

$$
\left\langle x^{k+1}-\left(u^{k}-\gamma_{k} A^{*}\left(A u^{k}-B v^{k}\right)\right), x^{k+1}-x^{*}\right\rangle \leqslant 0 .
$$

Therefore,

$$
\begin{aligned}
\left\langle x^{k+1}-u^{k}, x^{k+1}-x^{*}\right\rangle & \leqslant \gamma_{k}\left\langle B v^{k}-A u^{k}, A x^{k+1}-A x^{*}\right\rangle \\
& \leqslant \gamma_{k}\left\|A u^{k}-B v^{k}\right\|\left\|A x^{k+1}-A x^{*}\right\| \\
& \leqslant \delta\left\|A u^{k}-B v^{k}\right\|\left\|A x^{k+1}-A x^{*}\right\|
\end{aligned}
$$

which, combining with (3.22), implies that

$$
\lim _{k \rightarrow \infty}\left\langle x^{k+1}-u^{k}, x^{k+1}-x^{*}\right\rangle=0 .
$$

Likewise,

$$
\lim _{k \rightarrow \infty}\left\langle y^{k+1}-v^{k}, y^{k+1}-y^{*}\right\rangle=0 .
$$

From (3.20), (3.21), (3.26), (3.27), and (3.28), we have

$$
\lim _{k \rightarrow \infty}\left\|x^{k+1}-u^{k}\right\|^{2}+\left\|y^{k+1}-v^{k}\right\|^{2}=0
$$

Hence

$$
\lim _{k \rightarrow \infty}\left\|x^{k+1}-u^{k}\right\|=0
$$

and

$$
\lim _{k \rightarrow \infty}\left\|y^{k+1}-v^{k}\right\|=0
$$

According to the definition of $\theta_{k}$, we have

$$
\lim _{k \rightarrow \infty} \theta_{k}\left\|x^{k}-x^{k-1}\right\|=0
$$

and

$$
\lim _{k \rightarrow \infty} \theta_{k}\left\|y^{k}-y^{k-1}\right\|=0
$$

It follows from (3.29), (3.30), and the triangle inequality

$$
\left\|x^{k+1}-x^{k}\right\| \leqslant\left\|x^{k+1}-u^{k}\right\|+\left\|u^{k}-x^{k}\right\|=\left\|x^{k+1}-u^{k}\right\|+\theta_{k}\left\|x^{k}-x^{k-1}\right\|,
$$

one has

$$
\lim _{k \rightarrow \infty}\left\|x^{k+1}-x^{k}\right\|=0
$$

Analogous to the above proof, we can deduce

$$
\lim _{k \rightarrow \infty}\left\|y^{k+1}-y^{k}\right\|=0
$$

Because $\left\{x^{k}\right\}$ and $\left\{y^{k}\right\}$ are bounded sequences, there exist some weakly convergent subsequences, say $\left\{x_{i}^{k}\right\} \subset\left\{x^{k}\right\}$ and $\left\{y_{i}^{k}\right\} \subset\left\{y^{k}\right\}$ such that $x_{i}^{k} \rightarrow \bar{x}$ and $y_{i}^{k} \rightarrow \bar{y}$. According to (3.5), (3.30), and (3.31), we know that $u_{i}^{k} \rightarrow \bar{x}$ and $v_{i}^{k} \rightarrow \bar{y}$. Furthermore, from (3.5), we have $x^{k_{i}+1} \in C_{k_{i}}$. Therefore, by the definition of $\mathrm{C}_{\mathrm{k}_{\mathrm{i}}}$, one has

$$
c\left(x^{k_{i}}\right)+\left\langle\xi^{k_{i}}, x^{k_{i}+1}-x^{k_{i}}\right\rangle \leqslant 0
$$

where $\xi^{k_{i}} \in \partial c\left(x^{k_{i}}\right)$. Thus,

$$
c\left(x^{k_{i}}\right) \leqslant-\left\langle\xi^{k_{i}}, x^{k_{i}+1}-x^{k_{i}}\right\rangle \leqslant \xi\left\|x^{k_{i}+1}-x^{k_{i}}\right\|,
$$


where $\xi$ satisfies $\left\|\xi^{k}\right\| \leqslant \xi$ for all $k$. By the lower semicontinuity of $c(x),(3.32)$ and (3.33), we obtain

$$
c(\bar{x}) \leqslant \liminf _{k \rightarrow \infty} c\left(x^{k}\right) \leqslant \lim _{k_{i} \rightarrow \infty} c\left(x^{k_{i}}\right) \leqslant 0,
$$

that is, $\bar{x} \in \mathrm{C}$.

Similarly, we can deduce that $\bar{y} \in Q$.

Now, we show that $A \bar{x}=B \bar{y}$. In fact, the lower semicontinuity of the norm and (3.22) imply that

$$
\|A \bar{x}-B \bar{y}\| \leqslant \liminf _{k \rightarrow \infty}\left\|A u^{k}-B v^{k}\right\| \leqslant \lim _{k_{i} \rightarrow \infty}\left\|A u^{k_{i}}-B v^{k_{i}}\right\|=0,
$$

that is, $A \bar{x}=B \bar{y}$. Hence $(\bar{x}, \bar{y}) \in \Gamma$.

Finally, we prove the uniqueness of the weak cluster points of $\left\{x^{k}\right\}$ and $\left\{y^{k}\right\}$. Assume that $(\hat{x}, \hat{y})$ are the other weak cluster points of $\left\{x^{k}\right\}$ and $\left\{y^{k}\right\}$, respectively. Without loss of generality, we assume that $x^{k} \rightarrow \bar{x}, y^{k} \rightarrow \bar{y}$, and $x^{k} \rightarrow \hat{x}, y^{k} \rightarrow \hat{y}$, as well as $(\bar{x}, \bar{y}) \neq(\hat{x}, \hat{y})$. Let $S_{k}(\bar{x}, \bar{y})=\left\|x^{k}-\bar{x}\right\|^{2}+\left\|y^{k}-\bar{y}\right\|^{2}$. Then

$$
\begin{aligned}
S_{k}(\bar{x}, \bar{y}) & =\left\|x^{k}-\bar{x}\right\|^{2}+\left\|y^{k}-\bar{y}\right\|^{2} \\
& =\left\|x^{k}-\hat{x}\right\|^{2}+\|\hat{x}-\bar{x}\|^{2}+2\left\langle x^{k}-\hat{x}, \hat{x}-\bar{x}\right\rangle+\left\|y^{k}-\hat{y}\right\|^{2}+\|\hat{y}-\bar{y}\|^{2}+2\left\langle y^{k}-\hat{y}, \hat{y}-\bar{y}\right\rangle \\
& =S_{k}(\hat{x}, \hat{y})+\|\hat{x}-\bar{x}\|^{2}+\|\hat{y}-\bar{y}\|^{2}+2\left\langle x^{k}-\hat{x}, \hat{x}-\bar{x}\right\rangle+2\left\langle y^{k}-\hat{y}, \hat{y}-\bar{y}\right\rangle .
\end{aligned}
$$

Taking limit on the two sides of (3.34) and according to $x^{k} \rightarrow \hat{x}, y^{k} \rightarrow \hat{y}$, we have

$$
S(\bar{x}, \bar{y})=S(\hat{x}, \hat{y})+\|\bar{x}-\hat{x}\|^{2}+\|\bar{y}-\hat{y}\|^{2} .
$$

Reversing the role of $(\bar{x}, \bar{y})$ and $(\hat{x}, \hat{y})$, we have

$$
S(\hat{x}, \hat{y})=S(\bar{x}, \bar{y})+\|\bar{x}-\hat{x}\|^{2}+\|\bar{y}-\hat{y}\|^{2} .
$$

From (3.35) and (3.36), one has

$$
\|\bar{x}-\hat{x}\|^{2}+\|\bar{y}-\hat{y}\|^{2}=0,
$$

which implies that $\bar{x}=\hat{x}$ and $\bar{y}=\hat{y}$. This completes the proof.

In the following, we modify Algorithm 3.1 and present a new algorithm, denoted by the modified relaxed inertial accelerated algorithm.

\section{Algorithm 3.3.}

Initialization: Take $x^{0}, x^{1} \in \mathrm{H}_{1}, \mathrm{y}^{0}, \mathrm{y}^{1} \in \mathrm{H}_{2}$ arbitrarily.

Iterative step: For $k \geqslant 1$, given the points $x^{k}, x^{k-1}, y^{k}, y^{k-1}$, the next iterative points $x^{k+1}, y^{k+1}$ are generated by

$$
\left\{\begin{array}{l}
u^{k}=x^{k}+\theta_{k}\left(x^{k}-x^{k-1}\right), \\
v^{k}=y^{k}+\theta_{k}\left(y^{k}-y^{k-1}\right), \\
x^{k+1}=\left(1-\alpha_{k}\right) u^{k}+\alpha_{k} P_{C_{k}}\left(u^{k}-\gamma_{k} A^{*}\left(A u^{k}-B v^{k}\right)\right), \\
y^{k+1}=\left(1-\alpha_{k}\right) v^{k}+\alpha_{k} P_{Q_{k}}\left(v^{k}+\gamma_{k} B^{*}\left(A u^{k}-B v^{k}\right)\right),
\end{array}\right.
$$

where $\theta_{k} \in[0,1), \alpha_{k} \in(0,1), \gamma_{k}$ is positive stepsize, and $C_{k}, Q_{k}$ are defined as (3.3) and (3.4), respectively.

Now, we give the convergence theorem of Algorithm 3.3.

Theorem 3.4. For any $k \geqslant 1$, choose parameter $\theta_{k} \in\left[0, \bar{\theta}_{k}\right]$ with $\bar{\theta}_{k}=\min \left\{\theta,\left(\max \left\{k^{2}\left\|x^{k}-x^{k-1}\right\|^{2}, k^{2} \| x^{k}-\right.\right.\right.$ $\left.\left.\left.x^{k-1}\left\|, k^{2}\right\| y^{k}-y^{k-1}\left\|^{2}, k^{2}\right\| y^{k}-y^{k-1} \|\right\}\right)^{-1}\right\}$, where $\theta \in[0,1), 0<\inf _{k \geqslant 0} \alpha_{k}=\alpha<1$, and

$$
\gamma_{k} \in\left(\epsilon, \min \left\{\delta,(1-\sigma) \frac{2\left\|A u^{k}-B v^{k}\right\|^{2}}{\left\|A^{*}\left(A u^{k}-B v^{k}\right)\right\|^{2}+\left\|B^{*}\left(A u^{k}-B v^{k}\right)\right\|^{2}}\right\}\right),
$$

where $\epsilon>0$ is small enough, $\sigma \in(0,1)$, and $\delta>\epsilon$. Then the sequence $\left\{\left(x^{k}, y^{k}\right)\right\}$ generated by Algorithm 3.3 converges weakly to a solution $(x, y)$ of $(1.1)$.

Proof. Following the proof of Theorem 3.2 and combining with Lemma 2.3, we can easily complete the proof, which is omitted here. 


\section{Numerical examples}

In this section, we verify the feasibility and efficiency of Algorithms 3.1 and 3.3 through two examples. The whole codes are written in Matlab R2012a. All the numerical results are carried out on a personal Lenovo Thinkpad computer with Intel(R) Core(TM) i7-6500U CPU 2.50GHz and RAM 8.00GB.

Example 4.1. Let

$$
A=\left(\begin{array}{ccc}
-2 & -1 & 3 \\
0 & 2 & 5 \\
2 & 0 & 2
\end{array}\right), \quad B=\left(\begin{array}{ccc}
3 & 4 & 2 \\
0 & 1 & -5 \\
4 & 2 & 0
\end{array}\right)
$$

$\mathrm{C}=\left\{x \in \mathrm{R}^{3} \mid \mathrm{x}_{2}^{2}+\mathrm{x}_{3}^{2}-4 \leqslant 0\right\}$, and $\mathrm{Q}=\left\{x \in \mathrm{R}^{3} \mid \mathrm{x}_{3}-\mathrm{x}_{1}^{2}-1 \leqslant 0\right\}$. Find $\mathrm{x} \in \mathrm{C}, \mathrm{y} \in \mathrm{Q}$ such that $\mathrm{A} \mathrm{x}=\mathrm{By}$.

Example 4.2. Let

$$
A=\left(\begin{array}{ccccc}
2 & -1 & 3 & 2 & 3 \\
1 & 2 & 5 & 2 & 1 \\
2 & 0 & 2 & 1 & -2 \\
2 & -1 & 0 & -3 & 5
\end{array}\right), \quad B=\left(\begin{array}{llll}
1 & 0 & 2 & 0 \\
0 & 1 & 0 & 5 \\
0 & 0 & 1 & 0 \\
8 & 0 & 0 & 1
\end{array}\right)
$$

$C=\left\{x \in R^{5} \mid x_{1}^{2}+x_{2}^{2}+x_{3}^{2}+x_{4}^{2}+x_{5}^{2}-0.25 \leqslant 0\right\}$, and $Q=\left\{x \in R^{4} \mid x_{1}+x_{2}+x_{3}+x_{4}+0.6 \leqslant 0\right\}$. Find $x \in C, y \in Q$ such that $\mathrm{Ax}=\mathrm{By}$.

In the experiments, we take $\theta=0.2, \sigma=0.8, \theta_{k}=\bar{\theta}_{k}, \alpha_{k}=\frac{7}{8}-\frac{1}{2 k}$. And the stopping criterion is $\left\|x_{k+1}-x_{k}\right\| \leqslant 10^{-5}$. The calculation of projection and subdifferential depends on the following two lemmas.

Lemma $4.3([11,16])$. For every $\mathrm{k} \geqslant 0$, let $\mathrm{x}^{\mathrm{k}} \in \mathrm{R}^{\mathrm{n}}, \mathrm{C}_{\mathrm{k}}$, and $\mathrm{Q}_{\mathrm{k}}$ be defined as in (3.3) and (3.4), respectively. Then for any $\mathrm{x} \in \mathrm{R}^{\mathrm{n}}$, we have

$$
P_{C_{k}}(x)= \begin{cases}x-\frac{c\left(x^{k}\right)+\left\langle\xi^{k}, x-x^{k}\right\rangle}{\left\|\xi_{k}\right\|^{2}} \xi_{k}, & \text { if } c\left(x^{k}\right)+\left\langle\xi^{k}, x-x^{k}\right\rangle>0, \\ x, & \text { otherwise, }\end{cases}
$$

and

$$
P_{Q_{k}}(y)= \begin{cases}y-\frac{q\left(y^{k}\right)+\left\langle\eta^{k}, y-y^{k}\right\rangle}{\left\|\eta_{k}\right\|^{2}} \eta_{k}, & \text { if } \mathrm{q}\left(y^{k}\right)+\left\langle\eta^{k}, y-y^{k}\right\rangle>0, \\ y, & \text { otherwise. }\end{cases}
$$

Lemma 4.4 ([18]). Suppose $\mathrm{h}: \mathrm{R}^{\mathrm{n}} \rightarrow \mathrm{R}$ is a finite convex function, then it is subdifferentiable everywhere and its subdifferentials are uniformly bounded on any bounded subset of $\mathrm{R}^{\mathrm{n}}$.

In the following tables and figures, we denote the relaxed CQ algorithm, the relaxed inertial accelerated algorithm, and the modified relaxed inertial accelerated algorithm by "RCQA", "RIAA", and "MRIAA", respectively. And we set " $k$ ", "s", and " $x^{* \prime \prime}$ and " $y$ " to express the number of iteration, CPU time in seconds, and the final solution, respectively. Init. denote the initial points. The numerical results can be seen from Tables 1-3 and Figures 1-6.

From Tables 1-3, we can see that the iterative number and CPU time of the relaxed inertial accelerated algorithm and the modified relaxed inertial accelerated algorithm are less than that of the relaxed CQ algorithm.

Furthermore, for testing the stationary property of iterative number, we carry out 500 experiments for different initial points which are presented randomly, such as

$$
\begin{aligned}
& x^{0}=y^{0}=\operatorname{ones}(3,1), x^{1}=y^{1}=\operatorname{rand}(3,1), \\
& x^{0}=y^{0}=\operatorname{ones}(3,1), x^{1}=y^{1}=\operatorname{rand}(3,1) * 10,
\end{aligned}
$$

separately in Example 4.1, the results can be found in Figures 1-3. 
Table 1: The numerical results of Example 4.1.

\begin{tabular}{|l|l|l|}
\hline \multirow{5}{*}{ Init. } & $x^{0}=(1,1,1)^{\top}$ & $x^{0}=(-5,2,-10)^{\top}$ \\
& $x^{1}=(0.7792,0.9340,0.1299)^{\top}$ & $x^{1}=(1.3607,8.6929,5.7970)^{\top}$ \\
& $y^{0}=(1,1,1)^{\top}$ & $y^{0}=(100,-8,300)^{\top}$ \\
& $y^{1}=(0.5688,0.4694,0.0119)^{\top}$ & $y^{1}=(5.4986,1.4495,8.5303)^{\top}$ \\
\hline \multirow{3}{*}{ RCQA } & $k=63, s=0.0159$ & $k=207, s=0.0214$ \\
& $x^{*}=(2.4932,-1.2767,1.4645)^{\top}$ & $x^{*}=(1.0411,-0.1930,-1.9907)^{\top}$ \\
& $y^{*}=(2.5556,-1.1535,-1.1846)^{\top}$ & $y^{*}=(1.3481,-3.6458,1.3387)^{\top}$ \\
\hline \multirow{3}{*}{ RIAA } & $k=37, s=0.0129$ & $k=40, s=0.0170$ \\
& $x^{*}=(0.2831,0.7101,0.2565)^{\top}$ & $x^{*}=(1.0510,1.9279,0.5260)^{\top}$ \\
& $y^{*}=(0.3103,-0.0810,-0.5568)^{\top}$ & $y^{*}=(1.1718,-0.7667,-1.4505)^{\top}$ \\
\hline \multirow{3}{*}{ MRIAA } & $k=31, s=0.0095$ & $k=30, s=0.0061$ \\
& $x^{*}=(0.3253,0.7416,0.3225)^{\top}$ & $x^{*}=(0.9721,1.9740,0.3210)^{\top}$ \\
& $y^{*}=(0.3511,-0.0545,-0.6300)^{\top}$ & $y^{*}=(1.1075,-0.9220,-1.2950)^{\top}$ \\
\hline
\end{tabular}

Table 2: The numerical results of Example 4.2.

\begin{tabular}{|c|c|}
\hline Init. & $\begin{array}{l}x^{0}=(1,1,1,1,1)^{\top} \\
x^{1}=(0.4184,0.8680,0.1086,0.0128,0.7041)^{\top} \\
y^{0}=(1,1,1,1)^{\top} \\
y^{1}=(0.0960,0.8834,0.7770,0.6840)^{\top}\end{array}$ \\
\hline RCQA & $\begin{array}{l}\mathrm{k}=179, \mathrm{~s}=0.0146 \\
x^{*}=(-0.3061,0.1304,-0.3008,-0.1866,-0.1184)^{\top} \\
y^{*}=(-0.0459,-0.0039,-1.1637,-0.4074)^{\top}\end{array}$ \\
\hline RIAA & $\begin{array}{l}\mathrm{k}=77, \mathrm{~s}=0.0113 \\
x^{*}=(-0.1069,0.3240,-0.1948,0.1697,-0.0336)^{\top} \\
y^{*}=(-0.1505,-0.0718,-0.3666,-0.0111)^{\top}\end{array}$ \\
\hline MRIAA & $\begin{array}{l}\mathrm{k}=65, \mathrm{~s}=0.0102 \\
x^{*}=(-0.1037,0.3546,-0.2223,0.2503,-0.0378)^{\top} \\
\mathrm{y}^{*}=(-0.1890,-0.0951,-0.3263,0.0104)^{\top}\end{array}$ \\
\hline
\end{tabular}

Table 3: The numerical results of Example 4.2.

\begin{tabular}{|c|c|}
\hline Init. & $\begin{array}{l}x^{0}=(-5,2,-10,8,-6)^{\top} \\
x^{1}=(0.6003,0.9131,0.8051,0.3314,0.5863)^{\top} \\
y^{0}=(100,-8,300,9)^{\top} \\
y^{1}=(0.6689,0.0742,0.5852,0.4875)^{\top}\end{array}$ \\
\hline RCQA & $\begin{array}{l}\mathrm{k}=275, \mathrm{~s}=0.0535 \\
x^{*}=(-0.2204,0.0519,-0.3540,-0.2448,-0.1162)^{\top} \\
y^{*}=(-0.0707,-3.6222,-1.1612,0.2260)^{\top}\end{array}$ \\
\hline RIAA & $\begin{array}{l}\mathrm{k}=52, \mathrm{~s}=0.0206 \\
x^{*}=(0.1269,0.3275,-0.1253,-0.0213,0.0681)^{\top} \\
\mathrm{y}^{*}=(0.0211,-0.6286,-0.1544,0.1619)^{\top}\end{array}$ \\
\hline MRIAA & $\begin{array}{l}\mathrm{k}=50, \mathrm{~s}=0.0040 \\
x^{*}=(0.1201,0.3676,-0.1231,-0.0071,0.0702)^{\top} \\
y^{*}=(0.0072,-0.6409,-0.1537,0.1874)^{\top}\end{array}$ \\
\hline
\end{tabular}



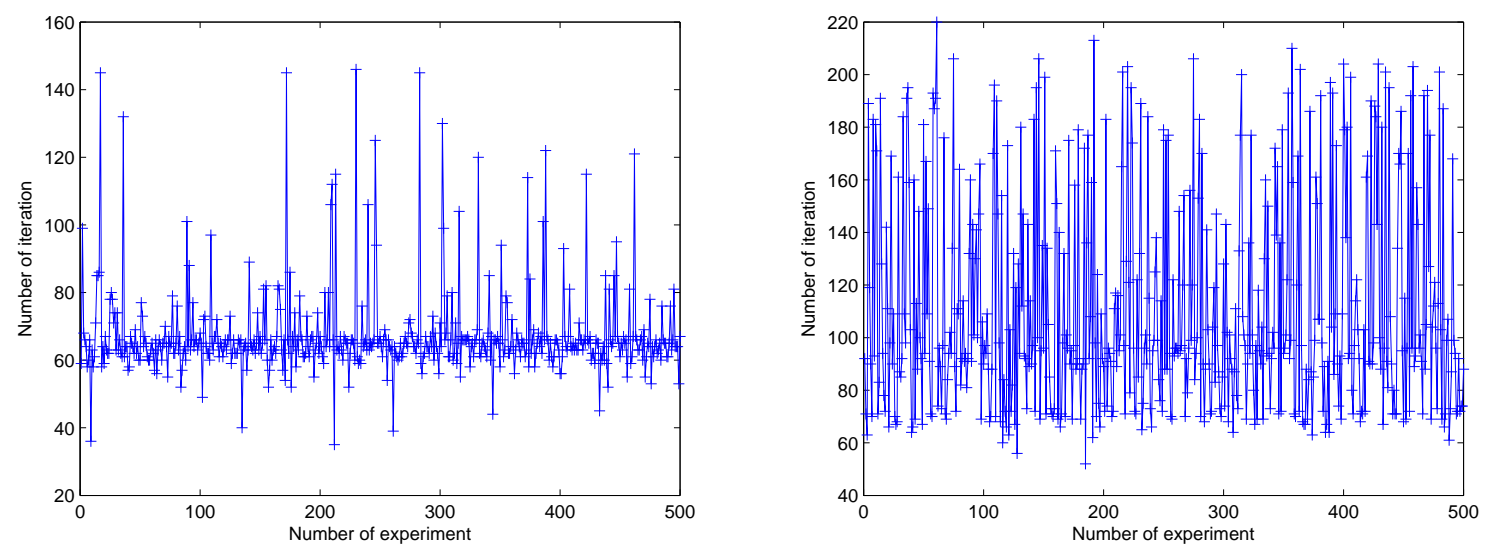

Figure 1: The iterative number of RCQA for Example 4.1.
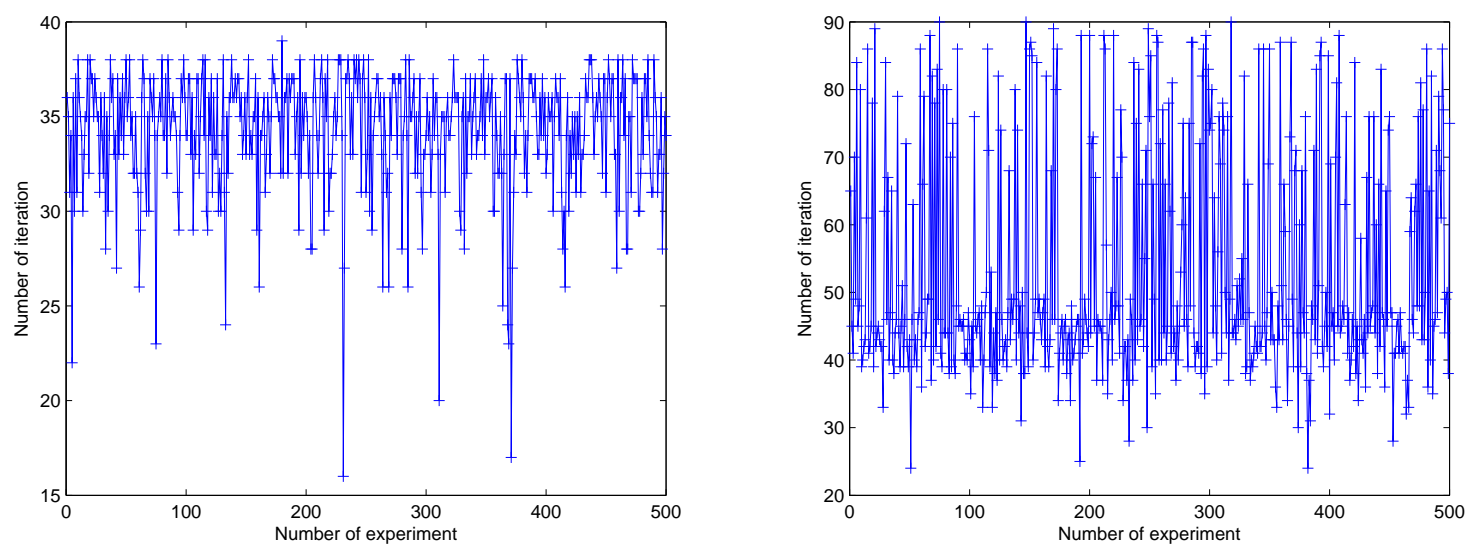

Figure 2: The iterative number of RIAA for Example 4.1.
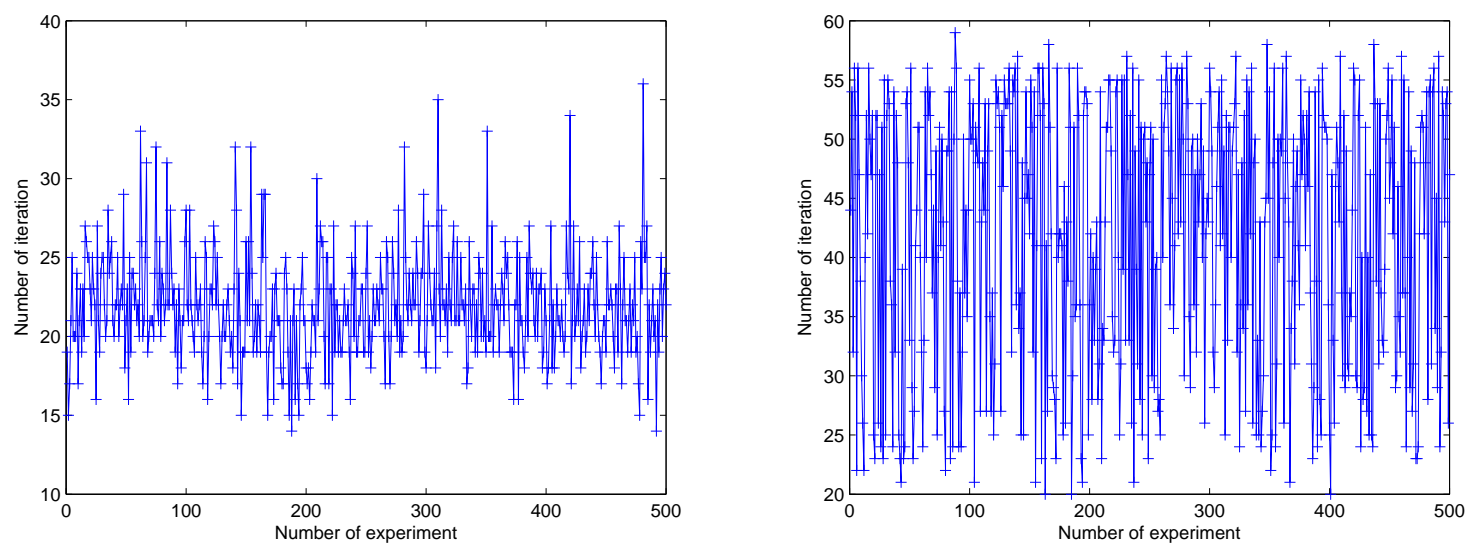

Figure 3: The iterative number of MRIAA for Example 4.1.

In the same way, we also perform 500 experiments for different initial points which are randomly presented in the following two cases

$$
\begin{aligned}
& x^{0}=\operatorname{ones}(5,1), y^{0}=\operatorname{ones}(4,1), x^{1}=\operatorname{rand}(5,1), y^{1}=\operatorname{rand}(4,1), \\
& x^{0}=\operatorname{ones}(5,1), y^{0}=\operatorname{ones}(4,1), x^{1}=\operatorname{rand}(5,1) * 10, y^{1}=\operatorname{rand}(4,1),
\end{aligned}
$$


separately in Example 4.2, the Figures 4-6 illustrate the results.
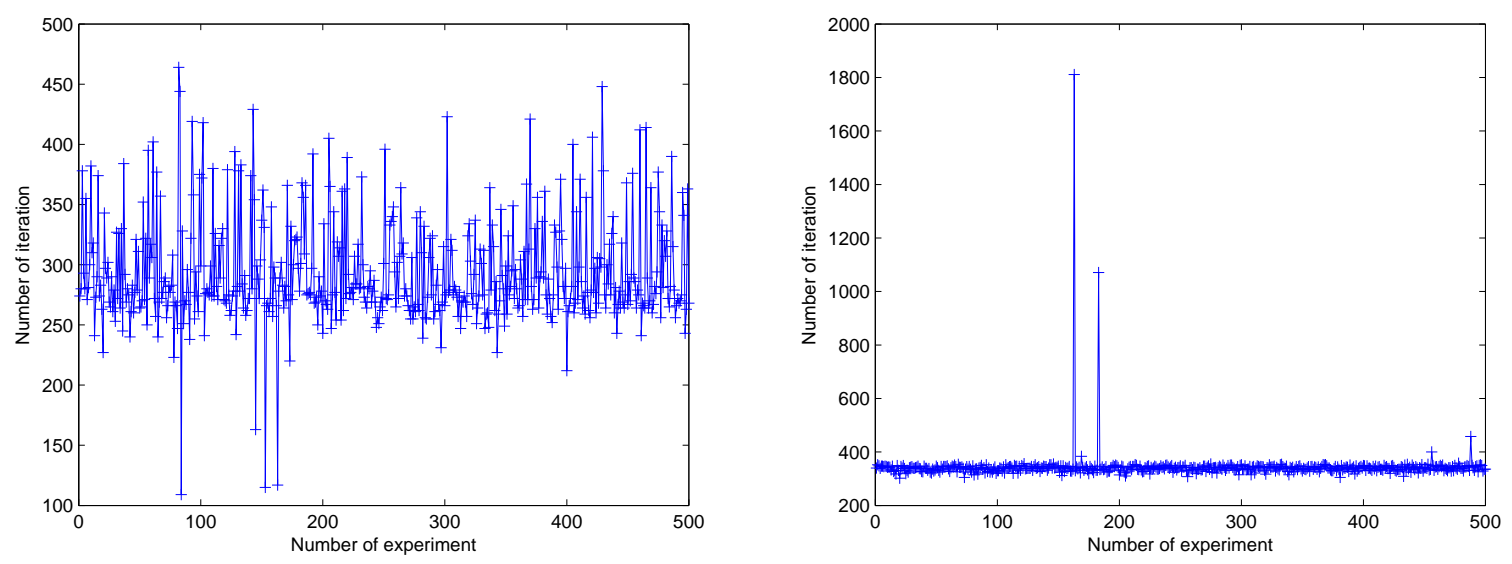

Figure 4: The iterative number of RCQA for Example 4.2.
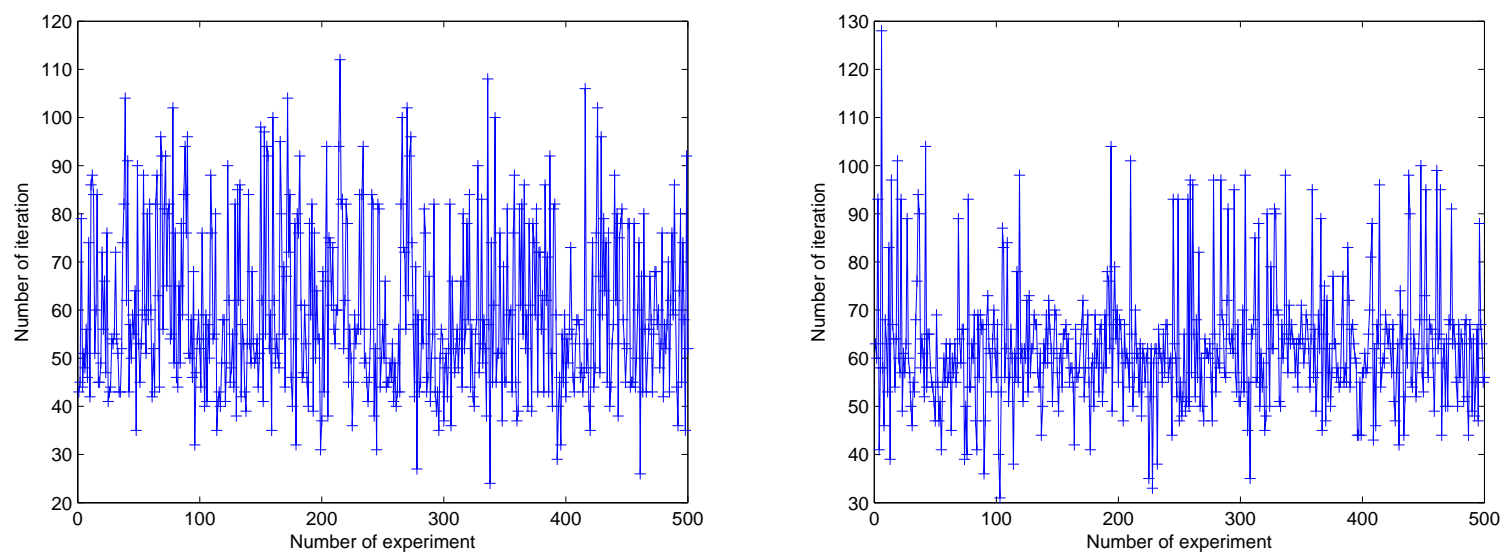

Figure 5: The iterative number of RIAA for Example 4.2.
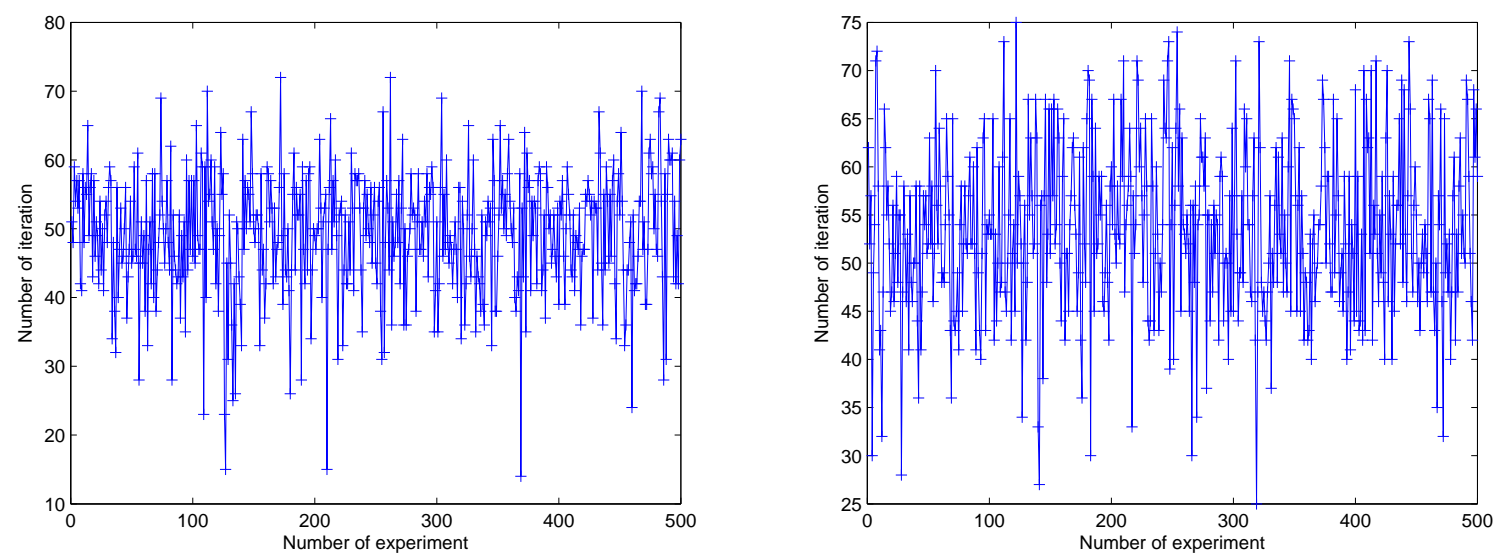

Figure 6: The iterative number of MRIAA for Example 4.2.

From Figures 1-6, we know that the iterative number of the three algorithms is stationary. Furthermore, 
we can see that the relaxed inertial accelerated algorithm and the modified relaxed inertial accelerated algorithm are superior than the relaxed CQ algorithm from the aspect of iterative number.

\section{Acknowledgment}

This research is supported by the Natural Science Foundation of China (no. 11401438, 11571120), Shandong Provincial Natural Science Foundation (no. ZR2013FL032), the Project of Shandong Province Higher Educational Science and Technology Program (no. J14LI52).

\section{References}

[1] H. H. Bauschke, J. M. Borwein, On projection algorithms for solving convex feasibility problems, SIAM Rev., 38 (1996), 367-426. 3

[2] C. L. Byrne, A. Moudafi, Extensions of the CQ Algorithm for the split feasibility and split equality problems (10th draft), hal-00776640-version 1, (2012). 1, 3

[3] Y. Censor, Parallel application of block-iterative methods in medical imaging and radiation therapy, Math. Programming, 42 (1998), 307-325. 1

[4] Y. Censor, T. Elfving, A multiprojection algorithm using Bregman projections in a product space, Numer. Algorithms, 8 (1994), 221-239. 1

[5] S.-S. Chang, Some problems and results in the study of nonlinear analysis, Proceedings of the Second World Congress of Nonlinear Analysts, Part 7, Athens, (1996), Nonlinear Anal., 30 (1997), 4197-4208. 2.3

[6] S.-S. Chang, L. Wang, L.-J. Qin, Split equality fixed point problem for quasi-pseudo-contractive mappings with applications, Fixed Point Theory Appl., 2015 (2015), 12 pages. 1

[7] H.-T. Che, M.-X. Li, A simultaneous iterative method for split equality problems of two finite families of strictly pseudononspreading mappings without prior knowledge of operator norms, Fixed Point Theory Appl., 2015 (2015), 14 pages. 1

[8] F. H. Clarke, Optimization and nonsmooth analysis, Canadian Mathematical Society Series of Monographs and Advanced Texts, A Wiley-Interscience Publication, John Wiley \& Sons, Inc., New York, (1983). 2.1

[9] Y.-Z. Dang, J. Sun, H.-L. Xu, Inertial accelerated algorithms for solving a split feasibility problem, J. Ind. Manag. Optim., 13 (2017), 1383-1394. 1

[10] F. Deutsch, The method of alternating orthogonal projections, Approximation theory, spline functions and applications, Maratea, (1991), NATO Adv. Sci. Inst. Ser. C Math. Phys. Sci., Kluwer Acad. Publ., Dordrecht, 356 (1992), $105-121$. 1

[11] M. Fukushima, A relaxed projection method for variational inequalities, Math. Programming, 35 (1986), 58-70. 3, 4.3

[12] Y. Gao, Piecewise smooth Lyapunov function for a nonlinear dynamical system, J. Convex Anal., 19 (2012), 1009-1015. 1

[13] G. T. Herman, Image reconstruction from projections: the fundamentals of computerized tomography, Academic Press, New York, (1980). 1

[14] P. E. Maingé, Convergence theorems for inertial KM-type algorithms, J. Comput. Appl. Math., 219 (2008), 223-236. 2.2

[15] A. Moudafi, Alternating CQ-algorithms for convex feasibility and split fixed-point problems, J. Nonlinear Convex Anal., 15 (2014), 809-818. 1

[16] B. T. Polyak, Minimization of unsmooth functionals, USSR Comput. Math. Math. Phys., 9 (1969), 14-29. 3, 4.3

[17] B. Qu, N.-H. Xiu, A new halfspace-relaxation projection method for the split feasibility problem, Linear Algebra Appl., 428 (2008), 1218-1229. 1

[18] R. T. Rockafellar, Convex analysis, Princeton Mathematical Series, No. 28 Princeton University Press, Princeton, N.J., (1970). 4.4

[19] H.-K. Xu, A variable Krasnoselskiü-Mann algorithm and the multiple-set split feasibility problem, Inverse Problems, 22 (2006), 2021-2034. 1

[20] A.-L. Yan, G.-Y. Wang, N.-H. Xiu, Robust solutions of split feasibility problem with uncertain linear operator, J. Ind. Manag. Optim., 3 (2007), 749-761.

[21] Q.-Z. Yang, The relaxed CQ algorithm solving the split feasibility problem, Inverse Problems, 20 (2004), 1261-1266.

[22] J.-L. Zhao, Q.-Z. Yang, Several solution methods for the split feasibility problem, Inverse Problems, 21 (2005), 1791-1799. 1 